\title{
Recruitment and retention of fathers with young children in early childhood health intervention research: a systematic review and meta-analysis protocol
}

Elizabeth M. Keys ${ }^{1}$, Jill M. Norris ${ }^{1}$, Emily E. Cameron ${ }^{2}$, Katherine S. Bright ${ }^{1}$, Lianne M. Tomfohr-Madsen ${ }^{2,3,4}$ and Karen M. Benzies ${ }^{1,3,4,5^{*}}$

\begin{abstract}
Background: Fathers are under-represented in research and programs addressing early childhood health and development. Recruiting fathers into these interventions can be hampered for multiple reasons, including recruitment and retention strategies that are not tailored for fathers. The primary aim of this systematic review and meta-analysis is to determine the effectiveness of recruitment and retention strategies used to include fathers of children (from conception to age 36 months) in intervention studies. The secondary aim is to investigate study-level factors that may influence recruitment and retention.

Methods: We will conduct searches for scholarly peer-reviewed randomized controlled trials, quasi-experimental studies, and pre-post studies that recruited fathers using the following databases: MEDLINE (Ovid), EMBASE (Ovid), PsycINFO (Ovid), and CINAHL. English-language articles will be eligible if they recruited self-identified fathers of children from conception to age 36 months for health-promoting interventions that target healthy parents and children. Two reviewers will independently screen titles/abstracts and full texts for inclusion, as well as grading methodological quality. Recruitment and retention proportions will be calculated for each study. Where possible, we will calculate pooled proportional effects with 95\% confidence intervals using random-effects models and conduct a meta-regression to examine the impact of potential modifiers of recruitment and retention.
\end{abstract}

Discussion: Findings from this review will help inform future intervention research with fathers to optimally recruit and retain participants. Identifying key factors should enable health researchers and program managers design and adapt interventions to increase the likelihood of increasing father engagement in early childhood health interventions. Researchers will be able to use this review to inform future research that addresses current evidence gaps for the recruitment and retention of fathers. This review will make recommendations for addressing key target areas to improve recruitment and retention of fathers in early childhood health research, ultimately leading to a body of evidence that captures the full potential of fathers for maximizing the health and wellbeing of their children.

Systematic review registration: PROSPERO CRD42018081332.

Keywords: Systematic review, Fathers, Infant, Father-child relations, Research subject recruitment, Intervention studies, Attrition

\footnotetext{
*Correspondence: benzies@ucalgary.ca

${ }^{1}$ Faculty of Nursing, University of Calgary, PF2278, 2500 University Drive NW,

Calgary, Alberta T2N $1 \mathrm{~N} 4$, Canada

${ }^{3}$ Alberta Children's Hospital Research Institute (ACHRI), Calgary, Alberta,

Canada

Full list of author information is available at the end of the article
}

C The Author(s). 2019 Open Access This article is distributed under the terms of the Creative Commons Attribution 4.0 International License (http://creativecommons.org/licenses/by/4.0/), which permits unrestricted use, distribution, and reproduction in any medium, provided you give appropriate credit to the original author(s) and the source, provide a link to the Creative Commons license, and indicate if changes were made. The Creative Commons Public Domain Dedication waiver (http://creativecommons.org/publicdomain/zero/1.0/) applies to the data made available in this article, unless otherwise stated. 


\section{Background}

Fathers contribute to the cognitive development, physical health, and social, emotional, and behavioral functioning of their children, over and above the contribution of mothers [1-8]. Fathers nurture, show affection, are responsive to their children's needs, and offer distinct parenting practices and experiences that differ from mothers [3, 6, 9-11]. Despite this acknowledgment, mothers remain the focus of research about young children, with fathers continuing to be under-represented in both research and programs addressing early childhood health and development [12-15]. For example, in a recent systematic review of early childhood sensitivity and attachment interventions that included 743 participants within 4 studies, only 2 participants $(<.01 \%)$ were male [16]. This dearth extends to observational research, with a systematic review of 667 studies noting only 17\% of the total participants were fathers [17]. Further, Rominov and colleagues' [18] systematic review found only 11 studies between 1994 and 2012 that examined father-focused mental health interventions. When compared to the maternal mental health intervention research described in several reviews of systematic reviews $[19,20]$, the notable difference between health research targeting mothers compared to fathers is reinforced, such that fathers are consistently a minority of the participant population.

Until the 1970s, fathers were rarely included in such research because societal norms and early theories of child development stressed the maternal role as primary caregiver with the belief that fathers had minimal direct influence on their children's development and functioning [13, $14,21]$. However, fathers have increasingly become more involved in their children's lives and care [4, 9, 21, 22]. Not only do fathers have a direct impact on their children's growth and development, but the support they provide for mothers is essential to enhance child outcomes $[4,22]$. In his review, Hoffman [22] concluded that "optimal support from the father can have a substantially positive impact on the mother's capacity for optimal caregiving and interaction with her baby" (p. 34). Yet, when compared to mothers, relatively little is known about effective interventions to help fathers support their partner and their children's development [23].

Recruiting fathers of children of any age to participate in research has proven challenging [24-27]. Potential obstacles to fathers' participation in child health and parenting interventions include not asking fathers to participate [28], maternal gatekeeping [29], work commitments, lack of time, and perceived gendered parenting roles [30]. In research examining father engagement in domestic violence prevention, the accessibility of programs (i.e., timing and location of services, costs, stigmatizing program names, and childcare availability), staff attitudes and behaviors, program marketing, and program format (i.e., use of groups, literacy level of media, and duration) were found to be potential contributors to father engagement [31]. Panter-Brick and colleagues [23] identified design and delivery conditions inherent to many parenting interventions that act as barriers to father engagement. Many barriers are modifiable and could be mitigated by increased awareness and father-inclusive design. Moreover, designing interventions that do not artificially isolate and address only the father-child relationship or the parenting couple's relationship could prove useful in creating an integrative approach whereby relational elements (fathering and parental/family relationships) are considered together [32]. Our experience in conducting observational studies suggests that many fathers of young children want to be involved in research [33-39]. In our intervention studies, fathers indicated that they were eager to learn about how to support their child's early development [40-42]. Thus, while there is overall support for early childhood health research becoming more father inclusive, as well as suggested target areas to address for engaging fathers, the evidence base examining the effectiveness of specific recruitment and retention methods has not yet been systematically analyzed.

\section{Objectives}

To support the ultimate goal of increasing the evidence base pertaining to interventions that help fathers support early childhood health, the objective of this review is to identify recruitment, retention, and research strategies to facilitate the participation of fathers of young children (from conception to age 36 months) in intervention research. The questions guiding this systematic review and meta-analysis are the following:

1. How effective are the recruitment and retention strategies used to include fathers (from conception to age 36 months) in intervention studies?

2. What are the potential study-level factors that influence recruitment and retention rates?

\section{Methods}

\section{Design}

We used the Preferred Reporting Items for Systematic reviews and Meta-analyses Protocols (PRISMA-P) statement [43] to guide this protocol (see Additional file 1) and have registered with PROSPERO CRD42018081332. We will document any amendments to the protocol with a rationale and report them with the final publication.

\section{Eligibility criteria Participants}

We will include studies of self-identified fathers of children from conception to age 36 months who participated in an intervention targeting medically and psychologically healthy 
parents and children. For this review, interventions that target medically or psychologically unwell individuals (i.e., mother, father, child) will be excluded. The rationale for this exclusion is that the underlying motivation of unwell individuals or family members of unwell individuals to engage in intervention research may be greater and/or specific based on the potential for additional treatment opportunities of an immediate concern. Thus, the review is limited to recruitment of fathers for health research that targets well individuals.

\section{Interventions}

We define an intervention as any educational or psychoeducational program that focuses on promoting health, wellbeing, or development of one or more family members, including the prevention of disease or health-harming behaviors. Interventions will include those targeted towards fathers, the family unit, mothers, or their child. Interventions for unwell individuals will be excluded, as per the participant inclusion criterion.

\section{Outcomes}

\section{Primary outcome}

Similar to other reviews of recruitment in intervention research $[44,45]$, recruitment for fathers will be calculated as a proportion of the number of fathers enrolled in research projects at baseline out of the total number of fathers identified as eligible.

\section{Secondary outcomes}

Additional recruitment proportions will be calculated based on previously described approaches to assessing recruitment $[46,47]$ that use, when available, (a) the total number of potential eligible individuals (pool), (b) the number of invited individuals from the pool (invited), (c) individuals who respond to the invitation to participate (responders), and (d) participants who were assessed as eligible and enrolled in the study (started). Recruitment rates will be calculated using previously described approaches [46-48] that calculate a rate based on the number of participants who started participation at each recruitment site divided by the total recruitment period (defined as the time in months between the start and completion of recruitment). Similar to Walters et al. [48], we will include proportions of actual to target sample sizes.

We will calculate retention based on a previously described strategy [49], in which the number of participants (fathers) retained in research projects until the time point of primary assessment outcome was collected is compared to the total number of participants enrolled. For studies with follow-up beyond the primary outcome collection time point, we will evaluate how many fathers were retained at each follow-up contact points. To calculate attrition rates, we will calculate the number of fathers lost during follow-up out of duration between follow-up time points.

\section{Study type}

Randomized controlled trials, quasi-experimental studies, and single group pre-post studies will be included in the review. Program evaluations that were not formalized intervention studies will be excluded. In cases of duplicate publications of the same sample, we will include the publication with the largest sample size. If sample sizes are consistent, the first published study from the sample will be included. We will exclude conference papers, dissertations, and other non-peer reviewed reports due to available resources for this review, the likelihood of these publication types having greater risk of unclear or high risk of bias. We will exclude reviews, as the primary focus of this review is on recruitment and retention methods used in primary research studies. Non-English publications will be excluded based on the study team's inability to translate non-English languages. The effect of excluding unpublished works, dissertations, and non-English reports is anticipated to be small [50].

\section{Information sources and search strategy}

MEDLINE (Ovid; 1946 to present), EMBASE (Ovid; 1974 to present), PsycINFO (Ovid; 1806 to present), and CINAHL (EBSCO; 1937 to present) databases will be searched. The search strategy was created with the assistance of an information scientist, and subject headings were modified to ensure they were database specific. The provisional MEDLINE search strategy is available in Table 1. In addition, we will conduct forward citation searches in Google Scholar, search the reference lists of included studies, and ask experts to identify further studies for inclusion. We will also hand search the reference lists of relevant systematic review articles. No filters will be used and no language restrictions will be placed. EndNote X8 will be used to manage the records, remove duplicates, and find full texts. We will re-run the searches immediately prior to the final analyses to retrieve any addition recently published articles for inclusion.

\section{Study selection}

Prior to screening titles and abstracts, we will conduct training and a calibration exercise with the review team to refine the screening tool in Microsoft Excel. Titles/abstracts will be independently screened by pairs of two reviewers. Disagreements will be resolved by a third reviewer. Full texts of potential studies will then be obtained and screened in the same manner with disagreements discussed as a review team. 
Table 1 Provisional search strategy

\begin{tabular}{|c|c|c|}
\hline 1 & exp Fathers/ & 7877 \\
\hline 2 & father.mp. & 41857 \\
\hline 3 & dad.mp. & 5999 \\
\hline 4 & dads.mp. & 759 \\
\hline 5 & paternal.mp. & 23160 \\
\hline 6 & or/1-5 & 65155 \\
\hline 7 & $\begin{array}{l}\text { exp Education, Nonprofessional/ } \\
\text { or exp Prenatal Education/ }\end{array}$ & 188637 \\
\hline 8 & psychoeducation*.mp. & 4021 \\
\hline 9 & psycho-education*.mp. & 1229 \\
\hline 10 & education*.tw,kf. & 486444 \\
\hline 11 & intervention*.mp. & 847036 \\
\hline 12 & program*.mp. & 877690 \\
\hline 13 & or/7-12 & 2010750 \\
\hline 14 & exp postpartum period/ & 59206 \\
\hline 15 & exp Labor, Obstetric/ & 45665 \\
\hline 16 & exp Labor, Induced/ & 9107 \\
\hline 17 & exp pregnancy/ & 853992 \\
\hline 18 & exp Parturition/ & 14311 \\
\hline 19 & exp infant/ & 1088414 \\
\hline 20 & postpartum.mp. & 61905 \\
\hline 21 & post-partum.mp. & 11505 \\
\hline 22 & postnatal.mp. & 99728 \\
\hline 23 & post-natal.mp. & 6709 \\
\hline 24 & antenatal.mp. & 31014 \\
\hline 25 & ante-natal.mp. & 516 \\
\hline 26 & perinatal.mp. & 67248 \\
\hline 27 & peri-natal.mp. & 184 \\
\hline 28 & pregnan*.mp. & 946625 \\
\hline 29 & birth*.mp. & 334360 \\
\hline 30 & childbirth.mp. & 18779 \\
\hline 31 & infancy.mp. & 59520 \\
\hline 32 & infant.mp. & 1136965 \\
\hline 33 & infants.mp. & 244504 \\
\hline 34 & toddler*.mp. & 9054 \\
\hline 35 & neonat*.mp. & 266522 \\
\hline 36 & baby.mp. & 34818 \\
\hline 37 & babies.mp. & 34051 \\
\hline 38 & prenatal.mp. & 157509 \\
\hline 39 & pre-natal.mp. & 1202 \\
\hline 40 & labor.mp. & 117454 \\
\hline 41 & labour.mp. & 28432 \\
\hline 42 & newborn*.mp. & 724924 \\
\hline 43 & or/14-42 & 2306627 \\
\hline 44 & 6 and 13 and 43 & 4681 \\
\hline
\end{tabular}

Table 1 Provisional search strategy (Continued)

\begin{tabular}{lll}
\hline 45 & prepartum.mp. & 2024 \\
46 & pre-partum.mp. & 313 \\
47 & 43 or 45 or 46 & 2306701 \\
48 & 6 and 13 and 47 & 4681 \\
\hline
\end{tabular}

Database(s): Ovid MEDLINE(R) Epub Ahead of Print, In-Process \& Other NonIndexed Citations, Ovid MEDLINE(R) Daily and Ovid MEDLINE(R) 1946 to present

\section{Data items and data extraction}

Table 2 details the data items to be extracted from the full texts, which are informed by the Template for Intervention Description and Replication (TIDieR) [51] and Transparent Reporting of Evaluations with Nonrandomized Designs (TREND) checklists [52]. Extracted data will include study characteristics, participants, intervention characteristics (including length and intensity), participant flow, assignment methods, recruitment methods, and retention methods. Data on recruitment and retention methods will be categorized and coded to better allow for assessment of how these methods impact recruitment and retention. We will use a templated data extraction tool in Microsoft Excel. Once the team completes a calibration exercise with the extraction tool, one reviewer will extract study data with a second reviewer verifying the extracted data. Discrepancies in extracted data will be resolved through discussion. Missing data or clarifications regarding ambiguous data will be requested by email from study authors.

\section{Study recruitment reporting quality}

The quality of recruitment reporting will be assessed using the grading scheme developed by Foster et al. [47], in which 2 independent reviewers will score 5 criteria related to the reporting of recruitment as a binary score, with scores of 0 used for criteria not reported or inadequately reported and a score of 1 allocated for criteria that are adequately described. Studies scoring 4 or 5 will be considered high quality for reporting recruitment, while studies scoring less than or equal to 3 will be considered low quality for reporting recruitment.

\section{Risk of bias in individual studies}

Studies will be included regardless of methodological quality. We will use the Cochrane risk of bias tool [53] to rate selection, performance, detection, attrition, reporting, and other biases in each study as either high, unclear, or low risk of bias. Two reviewers will independently assess all studies for quality, and disagreements will be resolved through discussion.

\section{Data synthesis}

Synthesis of the data will be conducted according to the Cochrane guidance [54]. Should the data permit, a metaanalysis of the recruitment and retention proportions 
Table 2 Data extraction categories for eligible studies

\begin{tabular}{ll}
\hline Category & Data extracted \\
\hline Study characteristics & First author, year, country, study design \\
Participant & $\begin{array}{l}\text { Eligibility criteria (including different levels in recruitment/sampling plan), } \\
\text { demographics, data collection setting }\end{array}$ \\
Intervention characteristics & $\begin{array}{l}\text { Content, delivery method, unit of delivery, deliverer, setting, exposure } \\
\text { quantity and duration, time span, effect size of intervention }\end{array}$ \\
Assignment method & $\begin{array}{l}\text { Unit of assignment } \\
\text { Method used to assign units to study conditions, including details of } \\
\text { any restriction } \\
\text { Inclusion of aspects employed to help minimize potential bias comparison }\end{array}$ \\
condition induced due to non-randomization \\
Participant flow & $\begin{array}{l}\text { Flow of participants: enrollment, assignment, allocation and intervention } \\
\text { exposure, baseline and outcome measures, follow-up }\end{array}$ \\
Recruitment & Method of recruitment, sampling method, recruitment setting \\
Retention & Method to increase compliance or adherence
\end{tabular}

will be conducted using Comprehensive Meta-Analysis (CMA) software, version 3.0. Heterogeneity of the studies' recruitment and retention proportions will be assessed by the non-parametric Cochran's $Q$ test, which assesses variance between studies and study populations. We will calculate the $I^{2}$ index to evaluate the proportion of heterogeneity between studies. If heterogeneity is present, random-effects models (as opposed to fixed-effect models) will be used as they are a more appropriate computational approach under conditions of heterogeneity given they are less likely to reject the null hypothesis. Further, random-effects models are more robust to large variations in sample sizes [55]. Recruitment and retention will be calculated as a proportional effect size with confidence intervals reported based on a $95 \%$ criterion. These proportions will be computed first as a proportional effect size and then transformed into logit units using CMA, which allows for more variability in the data than the bound proportional effect sizes [56, 57]. We will also conduct a sensitivity analysis by sequentially removing one study at a time and reanalyzing the dataset to determine the impact of any given single study. In conjunction with the quality analysis previously mentioned, this procedure allows for the inclusion of methodologically flawed studies if they meet this criterion. We will use a $\pm 5 \%$ range for acceptable, such that if one study affects the metaestimate by more than $\pm 5 \%$, it will be flagged for further consideration regarding inclusion in the meta-estimate. In addition, we will stratify the meta-analyses by high and low risk level of individual study bias, based on "high" ratings of bias for more than three of the six domains of bias examined by the Cochrane risk of bias tool and including a high level of risk for attrition bias. We will also stratify the meta-analyses by high and low recruitment reporting quality, based on a score of $\geq 4$ or $\leq 3$ on the quality of recruitment reporting grading scheme, respectively. Providing that there is sufficient information, we will also carry out meta-regression using logit units to explore potential modifiers of recruitment and retention rates, such as (a) study characteristics, (b) recruitment methods, (c) retention methods, (d) father and family characteristics, and (e) intervention characteristics. In the case that the included studies are too heterogeneous for meta-analysis, we will aggregate findings using narrative synthesis [58].

\section{Meta-bias}

Publication bias will be assessed through visual inspection of a funnel plot as well as statistical tests (e.g., Egger's regression intercept, Begg and Mazumdar's rank correlation, and Orwin's fail-safe $N$ ) [59-61].

\section{Confidence in cumulative evidence}

GRADE (Grades of Recommendation, Assessment, Development and Evaluation) [62] will be used to aid in evaluating the overall quality of the evidence in five domains: limitation of study design and execution, inconsistency, indirectness, imprecision, and publication bias. The overall quality of the evidence-rated as high, moderate, low, or very low-will be presented in a summary of findings table for each of the primary and secondary outcomes.

\section{Discussion}

To our knowledge, this protocol describes the first systematic review and meta-analyses that specifically examines the recruitment and retention rates of fathers in early childhood health intervention research. Previous reviews have described the lack of engagement of fathers in parenting interventions for children of all ages [23], domestic violence prevention [31], and obesity treatment and prevention research [63]. This systematic review will describe actual recruitment and retention strategies of early childhood health research, as well as the resulting recruitment and retention rates, including meta-analyses of participant and study characteristic subgroups. As such, we will be 
able to compare actual recruitment and retention rates with perceived facilitators and barriers [30, 64].

Our target knowledge users for our review findings are early childhood health intervention researchers and program managers. Accordingly, our dissemination strategies will target venues that researchers and program managers typically engage in to facilitate dissemination of findings. These venues include presentation of review results at local and national or international meetings, as well as publication in a peer-reviewed open-access journal. In addition, the research team will create and distribute a summary infographic, a promising visual knowledge translation tool [65], via social media, which has been reported to be used by health researchers and clinicians for professional purposes [66].

Limitations to the review may include limited data that may preclude the ability to run meta-analyses on all potential sub-groups of participant and study characteristics. Additionally, there may be limited descriptions of recruitment and retention strategies in the articles under review. Further, while articles may include fathers, data may only be presented on the couple and not disaggregated by mothers and fathers. Further limitations are related to the inclusion/exclusion criteria of reviewing only English-language articles, which may reduce generalizability to non-English speaking populations. Similarly, the inclusion of only peer-reviewed literature excludes government reports, dissertations, conference papers, and reviews thereby potentially increasing publication bias in our review and limiting grass-roots or community-based recruitment and retention strategies that may be used to target smaller or marginalized groups of fathers.

Findings from this review could help researchers design and develop more effective and evidence-based recruitment and retention strategies that enable and encourage father-inclusive early childhood health research. Managers of early childhood health programs who are interested in adopting a more father-inclusive approach may also be interested in using the review findings to consider how study-level factors may compare to program elements and promote or hinder father enrollment and retention. Identifying key facilitators and evidence gaps related to recruitment and retention of fathers in early childhood health intervention research should contribute to the overall body of evidence that aims to harness the potential of fathers in improving the health and wellbeing of their children.

\section{Supplementary information}

Supplementary information accompanies this paper at https://doi.org/10. 1186/s13643-019-1215-1.

Additional file 1. PRISMA-P checklist.

\section{Abbreviations}

GRADE: Grades of Recommendation, Assessment, Development and Evaluation; PRISMA-P: Preferred Reporting Items for Systematic reviews and Meta-analyses Protocols; TIDieR: Template for Intervention Description and Replication; TREND: Transparent Reporting of Evaluations with Nonrandomized Designs

\section{Acknowledgements \\ The authors are grateful to Joyce Magill-Evans for her vision and contributions to the initial version of this work. We thank Alix Hayden for assistance in creating the search strategy.}

\section{Authors' contributions}

KMB, LTM, EEC, EMK, and KSB conceived the review. All authors designed the protocol. EEC and KSB conducted the preliminary searches. All authors reviewed the manuscript and read and approved the final manuscript. KMB is the guarantor of the review.

\section{Funding}

EMK is supported by a Pre-Doctoral Canadian Child Health Clinician Scientist Program (CCHSP) Award, the Alberta Children's Hospital Research Institute (ACHRI)-Canadian Institute for Health Research (CIHR) Training Program, the Alberta Children's Hospital Foundation, and an Alberta Innovates - Health Solutions (AIHS) Clinician Fellowship. KSB is supported by the Graduate Studentship Award from the ACHRI and Faculty of Nursing, University of Calgary. EEC holds a Joseph-Armand Bombardier CGS Doctoral Scholarships from Social Sciences and Humanities Research Council (SSHRC). LTM holds a Career Development Award from CCHCSP and is supported by SSHRC (\#4302016-00469)

\section{Availability of data and materials}

The datasets created and analyzed during this review will be available from the corresponding author upon reasonable request. We will also deposit our datasets in an open access repository, Open Science Framework (https://osf. io/), developed and maintained by the Centre for Open Science.

Ethics approval and consent to participate

Not applicable.

\section{Consent for publication}

Not applicable.

\section{Competing interests}

The authors declare that they have no competing interests.

\section{Author details}

${ }^{1}$ Faculty of Nursing, University of Calgary, PF2278, 2500 University Drive NW, Calgary, Alberta T2N $1 \mathrm{~N} 4$, Canada. ${ }^{2}$ Department of Psychology, University of Calgary, Calgary, Alberta, Canada. ${ }^{3}$ Alberta Children's Hospital Research Institute (ACHRI), Calgary, Alberta, Canada. ${ }^{4}$ Department of Pediatrics, Cumming School of Medicine, University of Calgary, Calgary, Alberta, Canada. ${ }^{5}$ Department of Community Health Sciences, Cumming School of Medicine, University of Calgary, Calgary, Alberta, Canada.

Received: 7 May 2018 Accepted: 29 October 2019

Published online: 01 December 2019

\section{References}

1. Lamb ME. How do fathers influence children's development? Let me count the ways. In: Lamb ME, editor. The role of the father in child development. 5th ed. Hoboken: Wiley; 2010. p. 1-26.

2. Sarkadi A, Kristiansson R, Oberklaid F, Bremberg S. Fathers' involvement and children's developmental outcomes: a systematic review of longitudinal studies. Acta Paediatr. 2008;97(2):153-8.

3. Lewis C, Lamb ME. Fathers' influences on children's development: the evidence from two-parent families. Eur J Psychol Educ. 2003;18(2):211-28.

4. Yogman M, Garfield CF, Committee on Pyschosocial Aspects of Child and Family Health. Fathers' roles in the care and development of their children: the role of pediatricians. Pediatrics. 2016;138(1):e20161128.

5. Flouri E, Buchanan A. Early father's and mother's involvement and child's later educational outcomes. Br J Educ Psychol. 2004;74(2):141-53. 
6. McBride BA, Mills G. A comparison of mother and father involvement with their preschool age children. Early Child Res Q. 1993;8(4):457-77.

7. Varghese C, Wachen J. The determinants of father involvement and connections to children's literacy and language outcomes: review of the literature. Marriage Fam Rev. 2016;52(4):331-59.

8. William HJ. A meta-analysis: the relationship between father involvement and student academic achievement. Urban Educ. 2014;50(4):387-423.

9. Doucet A. 'Estrogen-filled worlds': fathers as primary caregivers and embodiment. Sociol Rev. 2006;54(4):696-716.

10. Jia R, Kotila LE, Schoppe-Sullivan SJ. Transactional relations between father involvement and preschoolers' socioemotional adjustment. J Fam Psychol. 2012;26(6):848-57.

11. Cabrera NJ, Fitzgerald HE, Bradley RH, Roggman L. The ecology of fatherchild relationships: an expanded model. J Fam Theory Rev. 2014;6(4):336-54.

12. Boyd ST. Study of the father: research methods. Am Behav Sci. 1985;29(1):112-28.

13. Phares V, Lopez E, Fields S, Kamboukos D, Duhig AM. Are fathers involved in pediatric psychology research and treatment? J Pediatr Psychol. 2005;30(8):631-43.

14. Phares $\mathrm{V}$. Where's poppa? The relative lack of attention to the role of fathers in child and adolescent psychopathology. Am Psychol. 1992;47(5):656-64.

15. Macfadyen A, Swallow V, Santacroce S, Lambert H. Involving fathers in research. J Spec Pediatr Nurs. 2011;16(3):216-9.

16. Mountain G, Cahill J, Thorpe H. Sensitivity and attachment interventions in early childhood: a systematic review and meta-analysis. Infant Behav Dev. 2017;46(Suppl C):14-32.

17. Davison KK, Gicevic S, Aftosmes-Tobio A, Ganter C, Simon CL, Newlan S, Manganello JA. Fathers' representation in observational studies on parenting and childhood obesity: a systematic review and content analysis. Am J Public Health. 2016:106(11):e14-21.

18. Rominov H, Pilkington PD, Giallo R, Whelan TA. A systematic review of interventions targeting paternal mental health in the perinatal period. Infant Ment Health J. 2016;37(3):289-301.

19. Alderdice F, McNeill J, Lynn F. A systematic review of systematic reviews of interventions to improve maternal mental health and well-being. Midwifery. 2013;29(4):389-99.

20. Barlow J, McMillan AS, Kirkpatrick S, Ghate D, Barnes J, Smith M. Health-led interventions in the early years to enhance infant and maternal mental health: a review of reviews. Child Adolesc Mental Health. 2010;15(4):178-85.

21. Lamb ME. The history of research on father involvement: an overview. Marriage Fam Rev. 2000;29(2-3):23-42

22. Hoffman J. Father factors: what social science research tells us about fathers and how to work with them. Father Involvement Research Alliance: Peterborough; 2011

23. Panter-Brick C, Burgess A, Eggerman M, McAllister F, Pruett $K$, Leckman JF. Practitioner review: engaging fathers - recommendations for a game change in parenting interventions based on a systematic review of the global evidence. J Child Psychol Psychiatry. 2014;55(11):1187-212.

24. Wong JJ, Roubinov DS, Gonzales NA, Dumka LE, Millsap RE. Father enrollment and participation in a parenting intervention: personal and contextual predictors. Fam Process. 2013;52(3):440-54.

25. Denzmore P, Dilorio C, McCarty F. The P.A.T.I.E.N.C.E. model: an approach for recruiting African American fathers and sons into behavioral research studies. Challenge (Atlanta Ga). 2005;11(2):38-54.

26. Wigfall VG, Brannen J, Mooney A, Parutis V. Finding the right man: recruiting fathers in inter-generational families across ethnic groups. Qual Res. 2013; 13(5):591-607.

27. Huebner RA, Werner M, Hartwig S, White S, Shewa D. Engaging fathers: needs and satisfaction in child protective services. Adm Soc Work. 2008;32(2):87-103.

28. Davison KK, Charles JN, Khandpur N, Nelson TJ. Fathers' perceived reasons for their underrepresentation in child health research and strategies to increase their involvement. Matern Child Health J. 2017;21(2):267-74.

29. Doyle O, Weller BE, Daniel SS, Mayfield A, Goldston DB. Overcoming barriers to fathers' participation in clinically relevant research: recommendations from the field. Soc Work Res. 2016:40(4):260-4

30. Tully LA, Collins DAJ, Piotrowska PJ, et al. Examining Practitioner Competencies, Organizational Support and Barriers to Engaging Fathers in Parenting Interventions. Child Psychiatry Hum Dev. 2018;49(1):109-22. https://doi.org/10.1007/s10578-017-0733-0.

31. Pfitzner N, Humphreys C, Hegarty K. Engaging men: a multi-level model to support father engagement. Child Fam Soc Work. 2017;22(1): 537-47.
32. Pruett MK, Pruett K, Cowan CP, Cowan PA. Enhancing father involvement in low-income families: a couples group approach to preventive intervention. Child Dev. 2017:88(2):398-407.

33. Harrison MJ. A comparison of parental interactions with term and preterm infants. Res Nurs Health. 1990;13(3):173-9.

34. Benzies KM. Relationship of early family environment to child behavioural development at age 7 years (Unpublished doctoral dissertation). Edmonton: University of Alberta; 2001.

35. Benzies KM, Harrison MJ, Magill-Evans J. Impact of marital quality and parent-infant interaction on preschool behavior problems. Public Health Nurs. 1998;15(1):35-43.

36. Boechler V, Harrison MJ, Magill-Evans J. Father-child teaching interactions: the relationship to father involvement in caregiving. J Pediatr Nurs. 2003; 18(1):46-51.

37. Magill-Evans J, Harrison MJ. Parent-child interactions and development of toddlers born preterm. West J Nurs Res. 1999;21(3):292-312.

38. Magill-Evans J, Harrison MJ. Parent-child interactions, parenting stress, and developmental outcomes at 4 years. Child Health Care. 2001;30(2):135-50.

39. Cameron EE, Hunter D, Sedov ID, Tomfohr-Madsen LM. What do dads want? Treatment preferences for paternal postpartum depression. J Affect Disord. 2017;215:62-70.

40. Magill-Evans J, Harrison MJ, Benzies K, Gierl M, Kimak C. Effects of parenting education on first-time fathers' skills in interactions with their infants. Fathering. 2007;5(1):42-57.

41. Benzies KM, Magill-Evans J, Harrison MJ, MacPhail S, Kimak C. Strengthening new fathers' skills in interaction with their 5-month-old infants: who benefits from a brief intervention? Public Health Nurs. 2008;25(5):431-9.

42. Benzies KM, Magill-Evans J, Kurilova J, Nettel-Aguirre A, Blahitka L, LacazeMasmonteil T. Effects of video-modeling on the interaction skills of first-time fathers of late preterm infants. Infants Young Child. 2013;26(4):333-48.

43. Moher D, Shamseer L, Clarke M, Ghersi D, Liberati A, Petticrew M, Shekelle P, Stewart LA, Group P-P. Preferred reporting items for systematic review and meta-analysis protocols (PRISMA-P) 2015 statement. Syst Rev. 2015;4(1):1.

44. Trivedi RB, Szarka JG, Beaver K, Brousseau K, Nevins E, Yancy WS Jr, Slade A, Voils $\mathrm{Cl}$. Recruitment and retention rates in behavioral trials involving patients and a support person: a systematic review. Contemp Clin Trials. 2013;36(1):307-18

45. Treweek $S$, Lockhart $P$, Pitkethly $M$, Cook JA, Kjeldstrøm M, Johansen M, Taskila TK, Sullivan FM, Wilson S, Jackson C, et al. Methods to improve recruitment to randomised controlled trials: Cochrane systematic review and meta-analysis. BMJ Open. 2013;3(2):e002360.

46. Cooke $\mathrm{R}$, Jones $\mathrm{A}$. Recruiting adult participants to physical activity intervention studies using sport: a systematic review. BMJ Open Sport Exerc Med. 2017;3(1):e000231.

47. Foster CE, Brennan G, Matthews A, McAdam C, Fitzsimons C, Mutrie N. Recruiting participants to walking intervention studies: a systematic review. Int J Behav Nutr Phys Act. 2011;8:137.

48. Walters SJ, Bonacho dos Anjos Henriques-Cadby I, Bortolami O, Flight L, Hind D, Jacques RM, Knox C, Nadin B, Rothwell J, Surtees M, et al. Recruitment and retention of participants in randomised controlled trials: a review of trials funded and published by the United Kingdom Health Technology Assessment Programme. BMJ Open. 2017;7(3): e015276.

49. Brueton VC, Tierney JF, Stenning S, Meredith S, Harding S, Nazareth I, Rait G. Strategies to improve retention in randomised trials: a Cochrane systematic review and meta-analysis. BMJ Open. 2014;4(2):e003821.

50. Hartling L, Featherstone R, Nuspl M, Shave K, Dryden DM, Vandermeer B. Grey literature in systematic reviews: a cross-sectional study of the contribution of non-English reports, unpublished studies and dissertations to the results of meta-analyses in child-relevant reviews. BMC Med Res Methodol. 2017;17(1):64

51. Hoffmann TC, Glasziou PP, Boutron I, Milne R, Perera R, Moher D, Altman $D G$, Barbour $V$, Macdonald $H$, Johnston $M$, et al. Better reporting of interventions: template for intervention description and replication (TIDieR) checklist and guide. BMJ. 2014;348:g1687.

52. Des Jarlais DC, Lyles C, Crepaz N, Group T. Improving the reporting quality of nonrandomized evaluations of behavioral and public health interventions: the TREND statement. Am J Public Health. 2004;94(3):361-6.

53. Higgins JP, Altman DG, Gotzsche PC, Juni P, Moher D, Oxman AD, Savovic J, Schulz KF, Weeks L, Sterne JA, et al. The Cochrane Collaboration's tool for assessing risk of bias in randomised trials. BMJ. 2011;343:d5928. 
54. Higgins J, Green S (eds.): Cochrane handbook for systematic reviews of interventions. Version 5.1.0. 2011. [updated March 2011]: The Cochrane Collaboration. Available from http://handbook.cochrane.org/.

55. Cooper $\mathrm{H}$, Hedges $L V$, Valentine $J C$, editors. The handbook of research synthesis and meta-analysis. New York: Russell Sage Foundation; 2009.

56. Lipsey M, Wilson D. Practical meta-analysis. Thousand Oaks: Sage; 2001.

57. Snijders $T A B$, Bosker RJ. Multilevel analysis: an introduction to basic and advanced multilevel modelling. 2nd. ed. London: Sage; 2012.

58. Popay J, Roberts H, Sowden A, Petticrew M, Arai L, Rodgers M, Britten N. Guidance on the conduct of narrative synthesis in systematic reviews. Lancaster: ESRC Research Methods Programme; 2006.

59. Begg CB, Mazumdar M. Operating characteristics of a rank correlation test for publication bias. Biometrics. 1994:50:1088-101.

60. Egger M, Smith GD, Schneider M, Minder C. Bias in meta-analysis detected by a simple, graphical test. BMJ. 1997;315(7109):629-34.

61. Orwin RG. A fail-safe N for effect size in meta-analysis. J Educ Stat. 1983;8(2):157-9.

62. Guyatt GH, Oxman AD, Vist GE, Kunz R, Falck-Ytter Y, Alonso-Coello P, Schunemann HJ, Group GW. GRADE: an emerging consensus on rating quality of evidence and strength of recommendations. BMJ. 2008; 336(7650):924-6.

63. Morgan PJ, Young MD, Lloyd AB, Wang ML, Eather N, Miller A, Murtagh EM, Barnes AT, Pagoto SL. Involvement of fathers in pediatric obesity treatment and prevention trials: a systematic review. Pediatrics. 2017;139(2):e20162635.

64. Tully LA, Piotrowska PJ, Collins DAJ, Mairet KS, Black N, Kimonis ER, Hawes DJ, Moul C, Lenroot RK, Frick PJ, et al. Optimising child outcomes from parenting interventions: fathers' experiences, preferences and barriers to participation. BMC Public Health. 2017;17(1):550.

65. Ibrahim AM, Lillemoe KD, Klingensmith ME, Dimick JB. Visual abstracts to disseminate research on social media: a prospective, case-control crossover study. Ann Surg. 2017;266(6):e46-8.

66. Tunnecliff J, llic D, Morgan P, Keating J, Gaida JE, Clearihan L, Sadasivan S, Davies D, Ganesh S, Mohanty P, et al. The acceptability among health researchers and clinicians of social media to translate research evidence to clinical practice: mixed-methods survey and interview study. J Med Internet Res. 2015;17(5)::119.

\section{Publisher's Note}

Springer Nature remains neutral with regard to jurisdictional claims in published maps and institutional affiliations.

Ready to submit your research? Choose BMC and benefit from:

- fast, convenient online submission

- thorough peer review by experienced researchers in your field

- rapid publication on acceptance

- support for research data, including large and complex data types

- gold Open Access which fosters wider collaboration and increased citations

- maximum visibility for your research: over $100 \mathrm{M}$ website views per year

At $\mathrm{BMC}$, research is always in progress.

Learn more biomedcentral.com/submissions 